Pacific Journal of Mathematics

OPEN MAPPING THEOREMS FOR PROBABILITY MEASURES

LARRY QUIN EIFLER 


\title{
OPEN MAPPING THEOREMS FOR PROBABILITY MEASURES ON METRIC SPACES
}

\author{
LARRY Q. EIFLER
}

Let $S$ and $T$ denote complete separable metric spaces. Let $P(S)$ denote the collection of probability measures on $S$ and equip $P(S)$ with the weak topology. If $\varphi: S \rightarrow T$ is continuous and onto, then $\varphi$ induces a weakly continuous mapping $\varphi^{0}$ of $P(S)$ onto $P(T)$. We show that $\varphi^{0}$ is open in the weak topology if and only if $\varphi$ is open. However, $\varphi^{0}$ is always open in the norm topology. Let $K$ be a totally disconnected compact metric space and let $S^{K}$ denote the set of continuous mappings of $K$ into $S$. Then there exists a natural mapping $\pi$ of $P\left(S^{K}\right)$ into $P(S)^{K}$. Blumenthal and Corson have shown that $\pi$ is onto. We establish that $\pi$ is an open mapping in the weak topology.

1. Introduction. Let $S$ be a complete separable metric space and let $C(S)$ denote the algebra of bounded continuous real-valued functions on $S$. Let $M(S)$ denote the collection of Borel measures on $S$ which have finite total variation $\|\mu\|$. Given $f \in C(S)$ and $\mu \in M(s)$, set $\mu(f)=\int f(s) d \mu(s)$. The weak topology on $M(S)$ is the topology on $M(S)$ induced by $C(S)$. Thus, a neighborhood system at $\mu$ in $M(S)$ is given by sets of the form

$$
\begin{array}{r}
N_{\epsilon}\left(\mu ; f_{1}, \cdots, f_{n}\right)=\left\{\nu \in M(S):\left|(\mu-\nu) f_{\imath}\right|<\epsilon \text { for } i=1, \cdots, n\right\} \\
\text { where } \epsilon>0 \text { and } f_{1}, \cdots, f_{n} \in C(S) .
\end{array}
$$

Let $M^{+}(S)$ denote the non-negative measures and let $P(S)$ denote the probability measures in $M(S)$.

Our goal is to establish open mapping theorems for some naturally induced mappings between sets of probability measures. Let $\varphi$ be a continuous map of $S$ onto $T$ where $S$ and $T$ are complete separable metric spaces. Define $\varphi^{0}: M(S) \rightarrow M(T)$ by

$$
\varphi^{0} \mu(g)=\mu(g \circ \varphi) \text { for each } g \in C(T) \text {. }
$$

A result of P. A. Meyer [9, p. 126] shows that $\varphi^{0}$ maps $P(S)$ onto $P(T)$. We show that $\varphi^{0}$ is open in the weak topology if and only if $\varphi$ is open.

Let $K$ be a totally disconnected compact metric space and let $S^{K}$ 
denote the collection of continuous maps of $K$ into $S$. Given $f, g \in S^{K}$, set $D(f, g)=\max \{d(f(x), g(x)): x \in K\}$ where $d$ is the metric on $S$. Then $S^{K}$ is a complete separable metric space with respect to $D$. Given $f \in C(S)$ and $x \in K$, we may define a mapping $f_{x}: S^{K} \rightarrow \mathbf{R}$ by $f_{x}(g)=f(g(x))$ for each $g \in S^{K}$. Now define a mapping $\pi$ : $P\left(S^{K}\right) \rightarrow P(S)^{K}$ by

$$
(\pi \mu)_{x}(f)=\mu\left(f_{x}\right) \text { for each } f \in C(S) .
$$

One easily checks that $x \rightarrow(\pi \mu)_{x}$ is continuous in the weak topology and so one may consider the family $(\pi \mu)_{x}$ as a continuous family of marginals associated with $\mu$. Blumenthal and Corson [1] have shown that $\pi$ maps $P\left(S^{K}\right)$ onto $P(S)^{K}$. We show that $\pi$ is open in the weak topology.

2. The mapping $\varphi^{0}: P(S) \rightarrow P(T)$. Other than the interior mapping principle for $F$-spaces $[6$, p. 55] and its generalizations, there are few results in functional analysis on openness of mappings. For example, P. Cohen [4] has shown that if $T: \ell_{1} \times \ell_{1} \rightarrow \ell_{1}$ is a continuous bilinear mapping which is onto, then $T$ need not be open at $(0,0)$. If $\Omega$ is a compact subset of a Banach space $B$ and if the mapping $(x, y) \rightarrow \frac{1}{2}(x, y)$ is open on $\Omega \times \Omega$, then the set ex $(\Omega)$ of extreme points of $\Omega$ is closed. Our example below shows that the converse, which was left unresolved by Vesterstrom [10, p. 293], is false. However, convex averaging is open on $P(S)$ and this plays a crucial role in our results.

EXAMPLE 2.1. There exists a compact convex subset $\Omega$ of $\mathbf{R}^{4}$ such that the extreme points of $\Omega$ are closed and the midpoint mapping $(x, y) \rightarrow \frac{1}{2}(x, y)$ is not open on $\Omega \times \Omega$. Let $\Omega$ be the convex hull of $(0,1,0$, $0)$ and $(0,-1,0,0)$ and $\left(x, 0,1, x^{2}\right)$ and $\left(x, 0,-1, x^{2}\right)$ for $0 \leqq x \leqq 1$. The extreme points of $\Omega$ are the two points and two arcs described above. But, the midpoint mapping is not open since $(0,1,0,0)+(0,-1$, $0,0)=(0,0,0,0)$ and $u, v \in \Omega$ with $\frac{1}{2}(u+v)=\left(x, 0,0, x^{2}\right)$ where $x \neq 0$ implies $u$ and $v$ are of the form $\left(x, 0, \lambda, x^{2}\right)$ where $-1 \leqq \lambda \leqq 1$.

Let $S$ be a complete separable metric space. We recall here some topological properties of $P(S)$ and $M^{+}(S)$. Every measure $\mu$ in $P(S)$ is tight [8, p. 32], i.e., given $\epsilon>0$, there is a compact subset $F$ of $S$ such that $\mu(S \backslash F)<\epsilon$. The weak topology on $M^{+}(S)$ is topologically complete. Thus, we may consider $M^{+}(S)$ and $P(S)$ as complete separable metric spaces. By embedding $S$ is a countable product of unit intervals and using the fact that the unit ball in space of uniformly continuous functions on a totally bounded metric space is separable, we have the following result $[8$, p. 47]. 
LEMMA 2.2. Let $S$ be a complete separable metric space. There exist continuous real-valued functions $g_{1}, g_{2}, \cdots$ on $S$ such that $\left\|g_{n}\right\|_{\infty} \leqq 1$ for $n=1,2, \cdots$ and such that the metric $\rho$ defined on $M^{+}(S)$ by

$$
\rho(\mu, \nu)=\sum_{n=1}^{\infty} 2^{-n}\left|(\mu-\nu) g_{n}\right|
$$

is equivalent to the weak topology on $\mathrm{M}^{+}(S)$.

We now show that convex averaging is open on $M^{+}(S)$. But, first we establish a result on selecting weakly convergent measures. We write $\mu_{n} \rightarrow \mu$ if $\left(\mu_{n}\right)_{n=1}^{\infty}$ converges to $\mu$ in the weak topology.

Proposition 2.3. Let $\mu_{n}, \mu \in M^{+}(S)$ where $\mu_{n} \rightarrow \mu$. Assume $0 \leqq$ $\nu \leqq \mu$. Then there exists $0 \leqq \nu_{n} \leqq \mu_{n}$ for $n=1,2, \cdots$ such that $\nu_{n} \rightarrow \nu$.

Proof. Given $\epsilon>0$, there exists $g$ continuous on $S$ such that $0 \leqq g \leqq 1$ and $\rho(g \mu, \nu)<\epsilon$. Hence, we may choose $f_{n}$ continuous on $S$ such that $0 \leqq f_{n} \leqq 1$ and $f_{n} \mu \rightarrow \nu$. But $f_{n} \mu_{k} \rightarrow f_{n} \mu$ as $k \rightarrow \infty$. So there exist $n_{1} \leqq n_{2} \leqq \cdots$ such that $n_{k} \rightarrow \infty$ and $\nu_{k}=f_{n_{k}} \mu_{k} \rightarrow \nu$.

THEOREM 2.4. Let $S$ be a complete separable metric space. Let $0<\lambda<1$. The mapping $(\mu, \nu) \rightarrow \lambda \mu+(1-\lambda) \nu$ is open on $\mathrm{M}^{+}(S) \times$ $M^{+}(S)$ and is open on $P(S) \times P(S)$.

Proof. Fix $\mu, \nu \in M^{+}(S)$ and set $\omega=\lambda \mu+(1-\lambda) \nu$. Assume $\omega_{n} \rightarrow \omega$ where $\omega_{n} \in M^{+}(S)$. Since $\lambda \mu \leqq \omega$, there exist $\mu_{n} \in M^{+}(S)$ such that $\mu_{n} \rightarrow \lambda \mu$ and $0 \leqq \mu_{n} \leqq \omega_{n}$. Hence,

$$
\frac{1}{\lambda} \mu_{n} \rightarrow \mu \quad \text { and } \quad \frac{1}{1-\lambda}\left(\omega_{n}-\mu_{n}\right) \rightarrow \nu
$$

Thus, the mapping $(\mu, \nu) \rightarrow \lambda \mu+(1-\lambda) \nu$ is an open map of $M^{+}(S) \times$ $M^{+}(S)$ onto $M^{+}(S)$. One readily obtains that convex averaging is an open map of $P(S) \times P(S)$ onto $P(S)$.

Let $S$ and $T$ be complete separable metric spaces and let $\varphi: S \rightarrow T$ be continuous and onto. Then $\varphi$ induces a mapping $\varphi^{0}: M(S) \rightarrow M(T)$ defined by $\varphi^{0} \mu(g)=\mu(g \circ \varphi)$ for each $g \in C(T)$. As noted in $\$ 1, \varphi^{0}$ maps $P(S)$ onto $P(T)$. We examine the openness of $\varphi^{0}$ on $P(S)$ with respect to the weak topology and the norm topology.

THEOREM 2.5. Let $S$ and T be complete separable metric spaces and 
let $\varphi: S \rightarrow T$ be continuous and onto. Then $\varphi$ is open if and only if $\varphi^{0}$ : $P(S) \rightarrow P(T)$ is open with respect to the weak topology.

Proof. Assume $\varphi^{0}: P(S) \rightarrow P(T)$ is open in the weak topology. Fix $s_{0} \in S$ and set $t_{0}=\varphi\left(s_{0}\right)$. Assume $\varphi$ is not open at $s_{0}$. Then there exist $t_{n} \rightarrow t_{0}$ and $\epsilon>0$ such that $d\left(s_{0}, \varphi^{-1}\left(t_{n}\right)\right) \geqq \epsilon$ for $n=1,2, \cdots$. Choose $f \in C(S)$ such that $f\left(s_{0}\right)=1$ and $f=0$ on $\left\{s \in S: d\left(s, s_{0}\right) \geqq \epsilon\right\}$. Since $U=\left\{\mu \in P(S):\left|\left(\mu-\delta_{s_{0}}\right) f\right|<\epsilon\right\}$ is a weak neighborhood of $\delta_{s,}$, there exist $N$ and $\mu_{n} \in \mathcal{U}$ such that $\varphi^{0} \mu_{n}=\delta_{t_{n}}$ for $n \geqq N$. But $\mu_{n}(f)=0$ since $\varphi^{-1}\left(t_{n}\right)$ supports $\mu_{n}$ and so $\mu_{n} \notin \mathscr{U}$, a contradiction.

Assume $\varphi: S \rightarrow T$ is open. Fix $\mu \in P(S)$. Let $\epsilon>0$ and let $f_{1}, \cdots, f_{n}: S \rightarrow[0,1]$ be continuous. Set $\mathscr{V}=\left\{\nu \in P(S):\left|(\mu-\nu) f_{i}\right|<\epsilon\right.$ for $i=1, \cdots, n\}$. We must show that $\varphi^{0} \mathcal{V}$ is a neighborhood of $\varphi^{0} \mu$ in $P(T)$. Choose $\mu_{0}, \mu_{1}, \cdots, \mu_{m} \in P(S)$ and $\lambda_{0}, \lambda_{1}, \cdots, \lambda_{m}>0$ such that

(1) $\mu=\sum \lambda_{,} \mu_{\text {, }}$

(2) $\lambda_{0}<\epsilon$ and each of $\mu_{1}, \cdots, \mu_{m}$ has compact support

(3) the oscillation of $f_{1}$ on the support of $\mu$, is less than $\epsilon / 2$ for each $i=1, \cdots, n$ and $j=1, \cdots, m$.

Set $\mathscr{V}_{1}=\left\{\nu \in P(S):\left|\left(\nu-\mu_{1}\right) f_{i}\right|<\epsilon\right.$ for $\left.i=1, \cdots, n\right\}$. Clearly, we have $\lambda_{0} P(S)+\lambda_{1} \mathscr{V}_{1}+\cdots+\lambda_{m} \mathscr{V}_{m} \subseteq \mathscr{V}$. We claim that $\varphi^{0} \mathscr{V}_{\text {, }}$ is a weak neighborhood of $\varphi^{0} \mu_{\text {. }}$. For each $j=1, \cdots, m$ choose an open subset $U$, of $S$ containing the support of $\mu_{1}$ such that the oscillations of $f_{1}, \cdots, f_{n}$ on $U_{\text {, }}$ are less than $\epsilon / 2$. Then $V_{1}=\varphi\left(U_{1}\right)$ is an open subset of $T$ containing the support of $\nu_{l}=\varphi^{0} \mu_{1}$. It suffices to show that $\nu \in \varphi^{0}\left(\mathscr{V}_{1}\right)$ if $\nu\left(V_{1}\right)>$ $1-\epsilon / 2$ and $\nu \in P(T)$. Choose $\beta_{0} \in P(T)$ and $\beta \in P\left(V_{l}\right)$ such that

$$
\nu=\frac{\epsilon}{2} \beta_{0}+\left(1-\frac{\epsilon}{2}\right) \beta
$$

Choose $\alpha_{0} \in P(S)$ and $\alpha \in P\left(U_{1}\right)$ such that $\varphi^{0} \alpha_{0}=\beta_{0}$ and $\varphi^{0} \alpha=\beta$. We have

$$
\varphi^{0}\left[\frac{\epsilon}{2} \alpha_{0}+\left(1-\frac{\epsilon}{2}\right) \alpha\right]=\nu
$$

and for $i=1, \cdots, n$

$$
\left|\left[\mu_{l}-\frac{\epsilon}{2} \alpha_{0}-\left(1-\frac{\epsilon}{2}\right) \alpha\right] f_{l}\right| \leqq \frac{\epsilon}{2}\left|\left(\mu_{1}-\alpha_{0}\right) f_{l}\right|+\left|\left(\mu_{1}-\alpha\right) f_{l}\right|<\epsilon .
$$

But $\varphi^{0} \mathscr{V} \supset \lambda_{0} P(T)+\lambda_{1} \varphi^{0} \mathcal{V}_{1}+\cdots+\lambda_{m} \varphi^{0} \mathscr{V}_{m}$ and so by Theorem 2.4, $\varphi^{0} \mathcal{V}$ is a weak neighborhood of $\varphi^{0} \mu$. 
We next show that the mapping $\varphi^{0}$ is open in the norm topology.

THEOREM 2.6. Let $S$ and $T$ be complete separable metric spaces and let $\varphi: S \rightarrow T$ be continuous and onto. Then $\varphi^{0}: M^{+}(S) \rightarrow M^{+}(T)$ is norm open and hence, $\varphi^{0}: P(S) \rightarrow P(T)$ is norm open.

Proof. Fix $\mu \in M^{+}(S)$ and set $\nu=\varphi^{0} \mu$. Assume $\nu_{n} \rightarrow \nu$ in norm where $\nu_{n} \in M^{+}(T)$. Choose compact subsets $K_{1} \subset K_{2} \subset \cdots$ of $S$ such that $\mu\left(K_{n}\right) \rightarrow \mu(S)$. Set $\alpha_{n}=\mu \mid K_{n}$ and $\beta_{n}=\varphi^{0} \alpha_{n}$. Then $\beta_{n}$ has compact support and $\beta_{n} \rightarrow \nu$. Also, $\nu_{k} \wedge \beta_{n} \rightarrow \beta_{n}$ as $k \rightarrow \infty$. Hence, there exist $1=n_{1} \leqq n_{2} \leqq \cdots$ such that $n_{k} \rightarrow \infty$ and $\nu_{k} \wedge \beta_{n_{k}} \rightarrow \nu$. As shown in [5, Lemma 2.2], there exist $0 \leqq \mu_{k} \leqq \alpha_{n k}$ satisfying $\rho^{0} \mu_{k}=\nu_{k} \wedge \beta_{n k}$. Then $\mu_{k} \rightarrow \mu$ in norm. Choose $\gamma_{k} \in M^{+}(S)$ such that $\varphi^{0} \gamma_{k}=$ $\nu_{k}-\left(\nu_{k} \wedge \beta_{n_{k}}\right)$. Then $\left\|\gamma_{k}\right\| \rightarrow 0$ and so $\mu_{k}+\gamma_{k} \rightarrow \mu$. Hence, $\varphi^{0}$ is open in the norm topology at $\mu$.

Remark 2.7. The proof of the openness of $\varphi^{0}$ in the weak topology seems to break into the two parts (1) $\varphi^{0}$ is open at the extreme points of $P(S)$ and (2) convex averaging is open on $P(T)$. There should be a general theorem on the openness of affine maps between convex subsets equipped with a metric which would yield Theorem 2.5.

Conjecture. Let $E$ and $F$ be Banach spaces and let $(E)_{1}$ and $(F)_{1}$ denote the closed unit ball in $E$ and $F$ respectively. Let $T: E \rightarrow F$ be continuous and linear. If $T$ maps $(E)_{1}$ onto $(F)_{1}$ and if $(E)_{1}$ is strictly convex, then $T$ is an open map of $(E)_{1}$ onto $(F)_{1}$.

Note. Example 2.1 resolves a conjecture of Clausing and Magerl in [3, p. 76]. S. M. Chang [2] has extended Theorem 2.4 to averaging of continuous collections of probability measures.

3. The mapping $\pi: P\left(S^{K}\right) \rightarrow P(S)^{K}$. Let $S$ be a complete separable metric space and let $K$ be a totally disconnected compact metric space. Let $S^{K}$ denote the collection of continuous maps of $K$ into $S$. We equip $S^{K}$ with the metric $D(f, g)=\max \{d(f(x), g(x))$ : $x \in K\}$ where $d$ is the metric on $S$. Thus $S^{K}$ is a complete separable metric space. The space $P(S)$ can be equipped with a metric which is equivalent to the weak topology and with respect to which $P(S)$ is complete and separable. Thus, the space $P(S)^{K}$ denotes the continuous maps of $K$ into $P(S)$ and $P(S)^{K}$ is equipped with the topology of uniform convergence in the weak topology. There is a natural mapping of $P\left(S^{K}\right)$ into $P(S)^{K}$. Let $\mu \in P\left(S^{K}\right)$ and $x \in K$. If $U$ is a Borel subset of $S$, then $\mu_{x}(U)=\mu\left(\left\{g \in S^{K}: g(x) \in U\right\}\right)$ defines a probability measure $\mu_{x}$ on $S$. One recognizes the family $\left(\mu_{x}\right)_{x \in K}$ as a family of marginals 
associated with $\mu$. The measure $\mu_{x}$ may alternately be defined as follows. Given $f \in C(S)$ and $x \in K$, define $f_{x}: S^{K} \rightarrow \mathbf{R}$ by $f_{x}(g)=$ $f(g(x))$. If $\mu \in P\left(S^{K}\right)$ and $x \in K$, then $\mu_{x}(f)=\mu\left(f_{x}\right)$. This latter equation shows that the mapping $x \rightarrow \mu_{x}$ is continuous in the weak topology. We set $\pi \mu(x)=\mu_{x}$. Blumenthal and Corson [1] have shown that $\pi$ maps $P\left(S^{K}\right)$ onto $P(S)^{K}$. Although there is no natural way of pulling back elements of $P(S)^{K}$ to $P\left(S^{K}\right)$, we shall prove that $\pi$ is an open mapping. We begin by extending Prop. 2.3 to continuous collections of nonnegative measures.

Lemma 3.1. Let $S$ be a complete separable metric space and let $X$ be a compact Hausdorff space. Let $0<\lambda<1$ and let $\Phi, \Psi: X \rightarrow P(S)$ be continuous. Assume $\Phi_{x} \geqq \lambda \Psi_{x}$ for each $x \in X$. If $\Phi_{n}: X \rightarrow P(S)$ and $\Phi_{n} \rightarrow \Phi$ uniformly in the weak topology, then there exist continuous maps $\Psi_{n}: X \rightarrow P(S)$ such that $\Phi_{n} \geqq \lambda \Psi_{n}$ for $n=1,2, \cdots$ and $\Psi_{n} \rightarrow \Psi$ uniformly in the weak topology.

Proof. By Lemma 2.2, we may choose continuous maps $g_{1}, g_{2}, \cdots$ of $S$ into $[0,1]$ such that the metric $\rho$ on $P(S)$ defined by $\rho(\mu, \nu)=$ $\Sigma 2^{-n}\left|(\mu-\nu) g_{n}\right|$ is equivalent to the weak topology on $P(S)$. If $f \in$ $C^{+}(S)$ and if $\mu \in P(S)$, then we define a nonnegative measure $f \cdot \mu$ on $S$ by $(f \cdot \mu) g=\mu(f g)$ for each $g \in C(S)$. For each $p=1,2, \cdots$, choose a partition of unity $f_{1}^{p}, \cdots, f_{n_{p}}^{p}$ for $S$ such that each of $g_{1}, \cdots, g_{p}$ has oscillation less than $1 / p$ on the support of $f_{t}^{p}$ for $i=1, \cdots, n_{p}$. Pick $\epsilon_{p}>0$ satisfying $p \epsilon_{p} n_{p}=1$. Given $\Lambda: X \rightarrow P(S)$, define $\pi_{p}(\Lambda): X \rightarrow$ $M^{+}(S)$ by

$$
\pi_{p}(\Lambda)_{x}=\sum \frac{\Psi_{x}\left(f_{1}^{p}\right)}{\Phi_{x}\left(f_{\imath}^{p}+\epsilon_{p}\right)} f_{1}^{p} \cdot \Lambda_{x}
$$

Recall that $f_{i}^{p} \cdot \Lambda_{x}(g)=\Lambda_{x}\left(f_{i}^{p} g\right)$ for each $g \in C(S)$.

Setting $f_{t}=f_{t}^{p}$ and $\epsilon=\epsilon_{p}$, we have

$$
\pi_{p}\left(\Phi_{m}\right)_{x}\left(g_{k}\right)=\sum \frac{\Psi_{x}\left(f_{\imath}\right)}{\Phi_{x}\left(f_{\imath}+\epsilon\right)}\left(\Phi_{m}\right)_{x}\left(f_{\imath} g_{k}\right)
$$

where $x \in X$ and $1 \leqq k \leqq p$. Let $\alpha^{k}\left(\beta_{1}^{k}\right)$ denote the minimum (maximum) of $g_{k}$ over the support of $f_{l}$. Then $\beta_{\imath}^{k}-\alpha^{k}<1 / p$. Also,

$$
\sum \alpha_{i}^{k} \Psi_{x}\left(f_{t}\right) \leqq \Psi_{x}\left(g_{k}\right) \leqq \sum \beta_{i}^{k} \Psi_{x}\left(f_{i}\right)
$$




$$
1-\frac{1}{p}<\frac{\left(\Phi_{m}\right)_{x}\left(f_{i}+\epsilon\right)}{\Phi_{x}\left(f_{1}+\epsilon\right)}<1+\frac{1}{p} \quad \text { for } \quad m \geqq M
$$

For $m \geqq M$ and $1 \leqq k \leqq p$, we have

$$
\begin{aligned}
\pi_{p}\left(\Phi_{m}\right)_{x}\left(g_{k}\right)-\Psi_{x}\left(g_{k}\right) \\
\quad \leqq \sum \frac{\Psi_{x}\left(f_{i}\right)}{\Phi_{x}\left(f_{\imath}+\epsilon\right)} \beta_{i}^{k}\left(\Phi_{m}\right)_{x}\left(f_{i}\right)-\sum \alpha_{\imath}^{k} \Psi_{x}\left(f_{\imath}\right) \\
\quad \leqq \sum\left(\frac{1}{p}+\beta_{\imath}^{k}-\alpha_{\imath}^{k}\right) \Psi_{x}\left(f_{\imath}\right) \\
\quad<\frac{2}{p} .
\end{aligned}
$$

On the other hand, for $m \geqq M$ and $1 \leqq k \leqq p$, we have

$$
\begin{aligned}
\pi_{p}\left(\Phi_{m}\right)_{x}\left(g_{k}\right)-\Psi_{x}\left(g_{k}\right) \\
\quad \geqq \sum \frac{\Psi_{x}\left(f_{i}\right)}{\Phi_{x}\left(f_{\imath}+\epsilon\right)} \alpha_{i}^{k}\left(\Phi_{m}\right)_{x}\left(f_{\imath}\right)-\sum \beta_{\imath}^{k} \Psi_{x}\left(f_{i}\right) \\
\quad \geqq \sum \frac{\Psi_{x}\left(f_{\imath}\right)}{\Phi_{x}\left(f_{\imath}+\epsilon\right)} \alpha_{\imath}^{k}\left(\Phi_{m}\right)_{x}\left(f_{\imath}+\epsilon\right)-\sum \beta_{\imath}^{k} \Psi_{x}\left(f_{\imath}\right)-\frac{1}{\lambda p} \\
\geqq \sum \Psi_{x}\left(f_{\imath}\right) \alpha_{\imath}^{k}\left(1-\frac{1}{p}\right)-\sum \beta_{\imath}^{k} \Psi_{x}\left(f_{\imath}\right)-\frac{1}{\lambda p} \\
\geqq-\frac{2}{p}-\frac{1}{\lambda p}=-\frac{1}{p}\left(2+\frac{1}{\lambda}\right) .
\end{aligned}
$$

Hence, for $m \geqq M, \quad\left\|\left[\pi_{p}\left(\Phi_{m}\right)-\Psi\right]\left(g_{k}\right)\right\|_{x} \leqq(2+1 / \lambda) / p$ if $1 \leqq k \leqq$ p. Thus, we may choose $m_{1}<m_{2}<\cdots$ such that $\left\|\left[\pi_{p}\left(\Phi_{m}\right)-\Psi\right]\left(g_{k}\right)\right\| \leqq$ $(2+1 / \lambda) / p$ if $k \leqq p$ and $m \geqq m_{p}$. Setting $\Psi_{m}=\pi_{p}\left(\Phi_{m}\right)$ if $m_{p} \leqq m<m_{p+1}$ and $\Psi_{m}=\Phi_{m}$ if $m<m_{1}$, we have $\Psi_{m} \rightarrow \Psi$ uniformly in the weak topology and also, $\lambda \Psi_{m} \leqq \Phi_{m}$. One may now modify the $\Psi_{m}$ so that $\Psi_{m}: X \rightarrow P(S)$ and at the same time preserve the uniform convergence to $\Psi$ and the inequality $\lambda \Psi_{m} \leqq \Phi_{m}$.

We next show that convex averaging is open on $P(S)^{X}$.

LEMMA 3.2. Let $X$ be a compact Hausdorff space and assume $0<\lambda<1$. Let $\Phi, \Psi: X \rightarrow P(S)$ be continuous. If $\mathcal{U}$ and $\mathscr{V}$ are neighborhoods of $\Phi$ and $\Psi$ in $P(S)^{X}$ respectively, then $\lambda \mathscr{U}+(1-\lambda) \mathscr{V}$ is a neighborhood of $\lambda \Phi+(1-\lambda) \Psi$.

Proof. Let $\Lambda_{n} \rightarrow \lambda \Phi+(1-\lambda) \Psi$ where $\Lambda_{n}: X \rightarrow P(S)$ is continuous. Then there exist $\Phi_{n}: X \rightarrow P(S)$ such that $\Phi_{n} \rightarrow \Phi$ and $\lambda \Phi_{n} \leqq$ 
$\Lambda_{n}$. Then $1 /(1-\lambda)\left(\Lambda_{n}-\lambda \Phi_{n}\right) \rightarrow \Psi$. Hence, $\lambda \mathcal{U}+(1-\lambda) \mathcal{V}$ is a neighborhood of $\lambda \Phi+(1-\lambda) \Psi$.

We are now prepared to show that the "marginal" mapping $\pi$ of $P\left(S^{K}\right)$ onto $P(S)^{K}$ is an open map. In [5], this result was proved for the case $S$ is compact and $K$ is a two point space.

THEOREM 3.3. Let $S$ be a complete separable metric space and let $K$ be a totally disconnected compact metric space. Then $\pi: P\left(S^{K}\right) \rightarrow P(S)^{K}$ is open in the weak topology.

Proof. Let $\mu \in P\left(S^{K}\right)$. Fix continuous maps $G_{1}, \cdots, G_{m}$ of $S^{K}$ into $[0, \infty)$. Set $U=\left\{\nu \in P\left(S^{k}\right):\left|(\nu-\mu) G_{j}\right|<1\right.$ for $\left.j=1, \cdots, m\right\}$. We need to show that $\pi \mathscr{U}$ is a neighborhood of $\pi \mu$. There exist $\mu_{0}, \mu_{1}, \cdots, \mu_{n} \in P\left(S^{K}\right), \lambda_{0}, \lambda_{1}, \cdots, \lambda_{n}>0, \delta>0$ and $f_{1}, \cdots, f_{n} \in S^{K}$ such that $\mu=\sum \lambda_{i} \mu_{1}$ and (1) the support of $\mu_{i}$ is a compact subset of $N_{\delta}\left(f_{t}\right)=\left\{f \in S^{K}: D\left(f, f_{t}\right)<\delta\right\}$ and (2) the oscillation of $G_{l}$ is less than $1 / 2$ over $N_{2 \delta}\left(f_{i}\right)$ for each $i=1, \cdots, n$ and $j=1, \cdots, m$. Now set $U_{i}=$ $\left\{\nu \in P\left(S^{K}\right):\left|\left(\nu-\mu_{i}\right) G_{j}\right|<1\right.$ for $\left.j=1, \cdots, m\right\}$ for $i=1, \cdots, n$. Then $\lambda_{0} P\left(S^{K}\right)+\lambda_{1} \mathcal{U}_{1}+\cdots+\lambda_{n} \mathcal{U}_{n} \subseteq \mathcal{U}$. By Lemma 3.2, it remains to verify that $\pi U_{\imath}$ is a neighborhood of $\pi \mu_{i}$. Let $M$ be an upper bound for $G_{1}, \cdots, G_{m}$. Choose $x_{1}, \cdots, x_{p}$ and compact subsets $K_{1}, \cdots, K_{p}$ of $K$ such that $K$ is the disjoint union of $K_{1}, \cdots, K_{p}$ and $x_{l} \in K_{j}$ and $K_{j} \subseteq$ $N_{\delta}\left(x_{j}\right)=\left\{x: d\left(x, x_{j}\right)<\delta\right\}$ and such that $f_{t}\left(K_{l}\right) \subseteq N_{\delta}\left(f_{t}\left(x_{l}\right)\right)$ for each $i=$ $1, \cdots, n$ and $j=1, \cdots, p$. Now the support of $\pi \mu_{i}(x)$ is contained in $N_{2 \delta}\left(f_{i}\left(x_{l}\right)\right)$ when $x \in K_{r}$. Choose $0<\lambda<1$ such that $(1-\lambda) M<$ $1 / 2$. Consider the set $\mathscr{V}_{\imath}=\left\{\Phi \in P(S)^{K}: \exists \Psi \in P(S)^{K}\right.$ such that $\Phi \geqq \lambda \Psi$ and the support of $\Psi_{x}$ is contained in $N_{\delta}\left(f_{l}\left(x_{\jmath}\right)\right)$ whenever $\left.x \in K_{l}\right\}$. Then $\mathscr{V}_{\imath}$ is a neighborhood of $\pi \mu_{i}$. We claim that $\pi \mathcal{U}_{\imath} \supset \mathscr{V}_{\imath}$. Fix $\Phi \in \mathscr{V}_{\imath}$ and choose $\Psi \in P(S)^{K}$ such that $\Phi \geqq \lambda \Psi$ and the support of $\Psi_{x}$ is contained in $N_{\delta}\left(f_{t}\left(x_{l}\right)\right)$ whenever $x \in K_{j}$. Then $\Psi \mid K_{\text {, }}$ is a continuous mapping of $K_{j}$ into $P\left(N_{\delta}\left(f_{i}\left(x_{l}\right)\right)\right)$. By the result of Blumenthal and Corson [1], we can choose $\nu_{l} \in P\left(N_{\delta}\left(f_{l}\left(x_{l}\right)\right)^{K_{l}}\right)$ such that $\pi \nu_{l}=\Psi \mid K_{l}$. Set $\nu=$ $\nu_{1} \times \cdots \times \nu_{p}$. Then $\nu$ is a probability measure on $S^{K}$ and satisfies $\pi \nu=\Psi$. Now choose $\omega \in P\left(S^{K}\right)$ such that $\pi \omega=(\Phi-\lambda \Psi) / \lambda$. Then $\pi[\lambda \nu+(1-\lambda) \omega]=\Phi$. Finally, we check that $\lambda \nu+(1-\lambda) \omega$ belongs to $\mathcal{U}_{\imath}$. If $1 \leqq j \leqq m$, then

$$
\begin{aligned}
\mid(\lambda \nu & \left.+(1-\lambda) \omega-\mu_{i}\right) G_{l} \mid \\
& \leqq \lambda\left|\left(\nu-\mu_{i}\right) G_{\jmath}\right|+(1-\lambda)\left|\left(\omega-\mu_{i}\right) G_{\jmath}\right| \\
& \leqq \lambda / 2+(1-\lambda) M<1 .
\end{aligned}
$$

Thus, $\pi U_{i}$ is a neighborhood of $\pi \mu_{i}$. 
4. Marginals for $P\left(\Pi X_{\lambda}\right)$. Let $X_{\lambda}$ be a compact Hausdorff space for each $\lambda \in \Lambda$ and let $\pi_{\lambda}$ denote the projection of $\Pi X_{\lambda}$ onto $X_{\lambda}$. If $\mu$ is a probability measure on $\Pi X_{\lambda}$, then the family of probability measures $\left(\mu_{\lambda}\right)_{\lambda \in \Lambda}$, defined by $\mu_{\lambda}(E)=\mu\left(\pi_{\lambda}^{-1}(E)\right)$ for each Borel subset $E$ of $X_{\lambda}$, is the family of marginals associated with $\mu$. We next give an open mapping result for the mapping $\mu \rightarrow\left(\mu_{\lambda}\right)_{\lambda \in \Lambda}$ with respect to the norm topology.

THEOREM 4.1. Suppose $X_{\lambda}$ is a compact Hausdorff space for each $\lambda \in \Lambda$. Let $\alpha \in P\left(\Pi X_{\lambda}\right)$ and let $\left(\alpha_{\lambda}\right)_{\lambda \in \Lambda}$ be the family of marginals associated with $\alpha$. Assume $\left(\beta_{\lambda}\right)_{\lambda \in \Lambda}$ is a family of probability measures where $\beta_{\lambda} \in P\left(X_{\lambda}\right)$. Then there exists $\beta \in P\left(\Pi X_{\lambda}\right)$ such that $\left(\beta_{\lambda}\right)_{\lambda \in \Lambda}$ is the family of marginals associated with $\beta$ and $\|\alpha-\beta\| \leqq \sum\left\|\alpha_{\lambda}-\beta_{\lambda}\right\|$.

Proof. Let $\alpha \in P\left(\Pi X_{\lambda}\right)$ and let $\left(\alpha_{\lambda}\right)_{\lambda \in \Lambda}$ be the family of marginals associated with $\alpha$. Fix $\left(\beta_{\lambda}\right)_{\lambda \in \Lambda}$ in $\Pi P\left(X_{\lambda}\right)$. Choose $x_{\lambda} \in X_{\lambda}$ for each $\lambda \in \Lambda$. Given a finite subset $F=\left\{\lambda_{1}, \cdots, \lambda_{n}\right\}$ of $\Lambda$, let $\alpha_{F}$ denote the probability measure obtained from $\alpha$ by the natural projection of $\Pi X_{\lambda}$ onto $\prod_{i=1}^{n} X_{\lambda_{i}}$. The associated marginals of $\alpha_{F}$ are $\alpha_{\lambda_{1}}, \cdots, \alpha_{\lambda_{n}}$. By applying a result in [5, Thm. 2.2], there exists a probability measure $\beta_{F}$ on $\Pi X_{\lambda_{1}}$ with associated marginals $\beta_{\lambda_{1}}, \cdots, \beta_{\lambda_{n}}$ satisfying $\left\|\alpha_{F}-\beta_{F}\right\| \leqq$ $\sum\left\|\alpha_{\lambda_{1}}-\beta_{\lambda_{1}}\right\|$. Let $\delta_{F}$ denote the point mass measure at $\left(x_{\lambda}\right)_{\lambda \in \Lambda \mid F}$ in $\Pi_{\lambda \in \Lambda \backslash F} X_{\lambda}$. Then $\delta_{F} \times \alpha_{F}$ and $\delta_{F} \times \beta_{F}$ are probability measures on $\Pi X_{\lambda}$. The net $\delta_{F} \times \alpha_{F}$ converges to $\alpha$ in the weak* topology. Let $\beta$ be a weak* limit point of the net $\delta_{F} \times \beta_{F}$ in $P\left(\Pi X_{\lambda}\right)$. Then, $\beta$ has associated marginals $\left(\beta_{\lambda}\right)_{\lambda \in \Lambda}$. Also, $\|\alpha-\beta\| \leqq \sup _{F}\left\|\alpha_{F}-\beta_{F}\right\| \leqq$ $\Sigma\left\|\alpha_{\lambda}-\beta_{\lambda}\right\|$.

\section{REFERENCES}

1. R. M. Blumenthal and H. H. Corson, On continuous collections of measures, Ann. Inst. Fourier, Grenoble, 20 (1970), 193-199.

2. S. M. Chang, On continuous image averaging of probability measures, (submitted to Pacific J. Math.).

3. A. Clausing and G. Magerl, Generalized Dirichlet problems and continuous selections of representing measures, Math. Ann., 216 (1975), 71-78.

4. P. Cohen, A counter example to the closed graph theorem for bilinear maps, J. Functional Analysis, 16 (1974), 235-240.

5. S. Z. Ditor and L. Q. Eifler, Some open mapping theorems for measures, Trans. Amer. Math. Soc., 164 (1972), 287-293.

6. N. Dunford and J. T. Schwartz, Linear operators. Part I, Interscience, 1958.

7. L. Q. Eifler, Some open mapping theorems for marginals, Trans. Amer. Math. Soc., 211 (1975).

8. K. R. Parthasarathy, Probability Measures on Metric Spaces, Academic Press, 1967.

9. L. Schwartz, Radon Measures, Oxford University Press, 1973.

10. J. Vesterstrom, On open maps, compact convex sets, and operator algebras, J. London Math. Soc., (2), 6 (1973), 289-297.

Received January 27, 1976.

University of Missouri at Kansas City 




\section{Pacific Journal of Mathematics}

\section{Vol. 66, No. $1 \quad$ November, 1976}

Helen Elizabeth. Adams, Factorization-prime ideals in integral domains ............ Patrick Robert Ahern and Robert Bruce Schneider, The boundary behavior of Henkin's kernel.

Daniel D. Anderson, Jacob R. Matijevic and Warren Douglas Nichols, The Krull

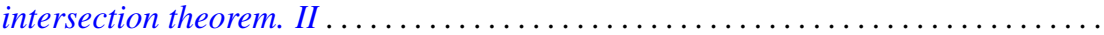

Efraim Pacillas Armendariz, On semiprime P.I.-algebras over commutative regular

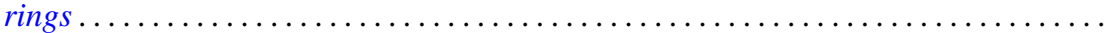

Robert H. Bird and Charles John Parry, Integral bases for bicyclic biquadratic fields

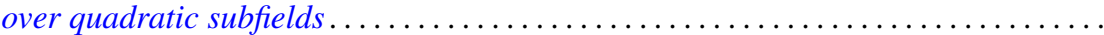

Tae Ho Choe and Young Hee Hong, Extensions of completely regular ordered

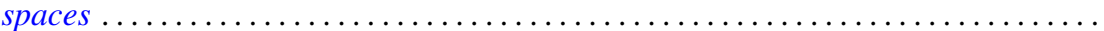

John Dauns, Generalized monoform and quasi injective modules ...............

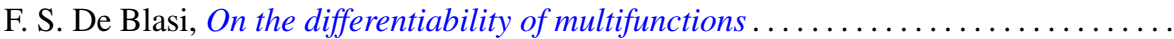

Paul M. Eakin, Jr. and Avinash Madhav Sathaye, R-endomorphisms of $R[[X]]$ are

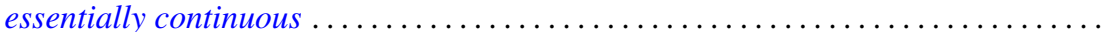

Larry Quin Eifler, Open mapping theorems for probability measures on metric

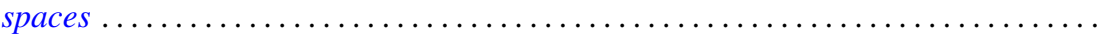

Garret J. Etgen and James Pawlowski, Oscillation criteria for second order self adjoint

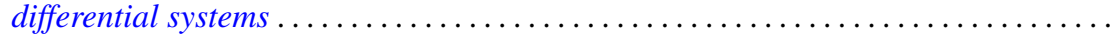

Ronald Fintushel, Local $S^{1}$ actions on 3-manifolds .

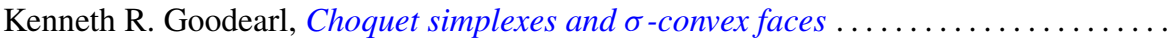

John R. Graef, Some nonoscillation criteria for higher order nonlinear differential

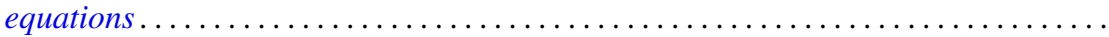

Charles Henry Heiberg, Norms of powers of absolutely convergent Fourier series: an example.

Les Andrew Karlovitz, Existence of fixed points of nonexpansive mappings in a space

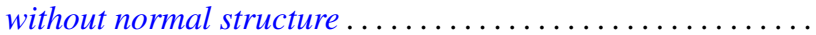

Gangaram S. Ladde, Systems of functional differential inequalities and functional

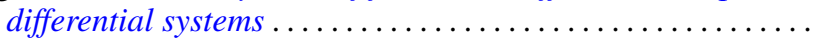

Joseph Michael Lambert, Conditions for simultaneous approximation and interpolation with norm preservation in $C[a, b]$.

Ernest Paul Lane, Insertion of a continuous function.

Robert F. Lax, Weierstrass points of products of Riemann surfaces .

Dan McCord, An estimate of the Nielsen number and an example concerning the Lefschetz fixed point theorem...

Paul Milnes and John Sydney Pym, Counterexample in the theory of continuous functions on topological groups...

Peter Johanna I. M. De Paepe, Homomorphism spaces of algebras of holomorphic functions

Judith Ann Palagallo, A representation of additive functionals on $L^{p}$-spaces,

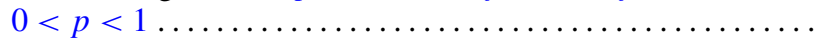

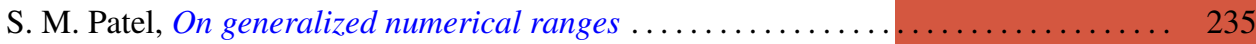

Thomas Thornton Read, A limit-point criterion for expressions with oscillatory

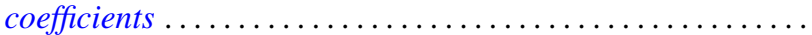

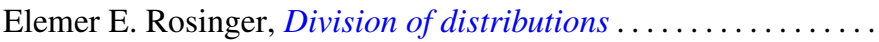

Peter S. Shoenfeld, Highly proximal and generalized almost finite

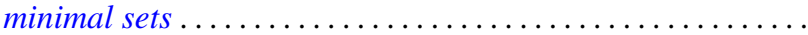

R. Sirois-Dumais and Stephen Willard, Quotient-universal sequential spaces

Robert Charles Thompson, Convex and concave functions of singular values of matrix sums....

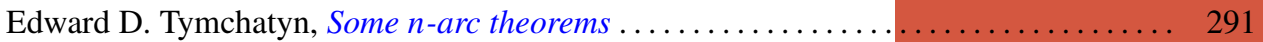

